\title{
CONVERGENCE
}

BY

MARGARET DOBSON

LONDON

Convergence may be summarised as consisting of three parts, tonic, accommodational, and fusional (Maddox). Tonic convergence is that amount of fusional innervation which is necessary to place the eyes in the position of parallelism at infinity.

The usual infinity distance in practice is six metres, at this distance actually $0 \cdot 16$ dioptres of accommodation and one prism dioptre of convergence are in use. Although Maddox suggested the term "Tonic" there is no reason for regarding this type of convergence as other than fusional, the same fusion innervation acts at distance as at the near point.

Accommodational convergence results from, and is associated with, any accommodational effort. These two functions are interconnected; contraction of the ciliary muscles is accompanied by a known and measurable amount of convergence. The contraction of the ciliaries may be insufficient or too great, but convergence must be exact for any point within infinity, to avoid diplopia. At the near point (33 cms.) eighteen prism dioptres are required, and as accommodation is variable, the final adjustment is known as fusional convergence.

If accommodational convergence is insufficient fusional convergence is positive (adductive), if it is excessive fusional convergence is negative (abductive).

All lateral muscle imbalances are errors of convergence, and all tests are convergence tests. Fusional convergence is the innervation, which corrects near point imbalances. In order to judge whether or not any-imbalance, either at distance or near, requires help to maintain efficiency, the exact fusional reserve which the patient possesses must be known.

The fusional amplitude can be ascertained by taking the patient's duction, which roughly represents the fusional amplitude, which may enable him to cope with the imbalance.

Any imbalance at the near point is always the difference between that measured at distance and that found at the near point (33 cms.). For example if three dioptres of exophoria is found at distance and five dioptres at near, the sum total of the near imbalance is two dioptres of exophoria, whereas if three dioptres of esophoria exists at distance and five dioptres of exophoria at near, the sum total will be eight dioptres of exophoria at the near point. 


\section{Physiological Exophoria}

A certain amount of exophoria at the reading distance, provided that it does not exceed six prism dioptres (P.D. $60 \mathrm{~mm}$.) is normal, and must be looked upon as a normal convergence lag due to the fact that accommodation is not exerted to the value of the fixation distance. In viewing ordinary print at $33 \mathrm{cms}$., we converge exactly eighteen prism dioptres; but three dioptres of accommodation are not in use, the amount is $2.05 \mathrm{D}$., or less, because average reading matter subtends a visual angle of not more than 6/12. Snellen.

This normal convergence lag is supplied by fusional convergence and indicates a normal shortage of accommodational effort.

Physiological exophoria permits a certain amount of hypermetropia to be tolerated without upsetting the muscle balance.

\section{Diagnosis of Convergent Imbalances}

In testing convergence imbalance we measure the tendency, relative convergence or divergence, of the visual axes at any point of fixation; and this should be done before and after the refractive error has been corrected, as often an accommodational convergence error is neutralised by correct lenses.

The imbalance in prism dioptres having been found, we ascertain if the opposing duction is able to cope with the error.

The following are four very useful tests which can be made to investigate the presence of insufficient or excessive convergence.

\section{The Cover Test}

This is perhaps the most accurate method for examination of any convergence imbalance. It is equally accurate for both distance and at near.

The ordinary dissociation tests, such as Maddox rod or wing test, or the procuring of diplopia by means of vertical prisms, though they prevent fusion, do not remove the desire to fuse, there is a tendency to align the diplopic images. The cover test not only prevents fusion, but removes the desire to fuse.

Snellen 6/9 line is used as a fixation target for distance, and the point of a pencil, for near.

In cases of established presbyopia the reading correction must be worn for testing at $33 \mathrm{cms}$. in addition to that necessary for distance.

The eyes fix the target, and an ebonite disc is placed over one eye, on removal an IN (exophoria) or an OUT (esophoria) movement of this eye is seen if any imbalance is present.

The imbalance can be accurately measured with base-in prisms 
(exophoria) and base-out prisms (esophoria), the strength of the prism which prevents movement being the measure of the imbalance. The actual position of the eye under cover can be seen, if a green (red free) light is used as a target. A polarised glass screen (the analyser) is placed in front of the light and a second screen (the catalyser) in front of the eye under examination, at right angles to the analyser.

The polarised screens prevent the patient from seeing the light, while the examiner can clearly see the position of the eye under the screen.

\section{The Prism Stereoscope Test}

This is a very reliable test for estimating convergence stability at the reading distance. Javal's F.L. card is placed at $33 \mathrm{cms}$. upon the bar of the stereoscope with the septum in such a position that $F$. is seen with the left eye, and L. with the right eye.
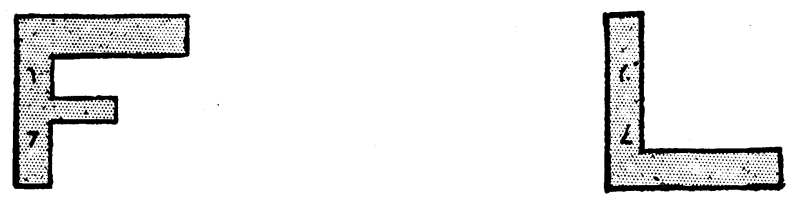

Nine prism dioptres are placed before each eye, with bases OUT, representing the normal amount of convergence in use for this distance, presuming that the P.D. is $60 \mathrm{~mm}$.

If convergence is normal the observer using both eyes sees $\mathrm{E}$. In cases of insufficient convergence $L$. is seen with the left eye and $F$. with the right eye, if over-convergence is present $F$. is seen with the left eye and $L$. with the right eye.

The prisms must be reduced in the case of insufficiency and increased in the case of over-convergence to obtain fusion.

The amount of exophoria or esophoria will be the difference between eighteen prism dioptres and the total amount of prism power which is required to form $\mathrm{E}$.

Small letters, which are seen as Y.Z. upon the vertical limb of the E. indicate a high grade binocular stability, in which the macula lutea is in use. If Y.Z. is seen, any degree of hyperphoria can be ruled out.

Convergence efficiency, must be determined by prism-convergence at $33 \mathrm{cms}$., the ordinary reading distance.

A test made with a small object or light as the fixation point, which is made to approach the end of the patient's nose, while the exact distance at which diplopia is noted, or the observer records 
deviation of one eye or displacement of the central light reflex of this eye, is loaded with inaccuracies.

Factors arise which do not occur at $33 \mathrm{cms}$.

Excessive accommodation on the approach of the object stimulates convergence, and the images on the retinae become enlarged, owing to shortening of the distance between the object and the nodal points of the eyes examined.

Convergence may also be stimulated by the fact that the patient is aware of the increasing nearness of the fixation point. In early presbyopia, which results in the receding of the near point, the object becomes blurred, this tends to inhibit fusion and to cause diplopia. For these reasons convergence efficiency or insufficiency cannot be diagnosed by this means.

These near imbalance tests measure the amount of fusional convergence in use.

A maximum of six prism dioptres of exophoria is a convergence lag representing a normal shortage of accommodation.

A greater amount of exophoria than normal is suggestive of insufficient convergence, while less suggests an increased accommodative effort due most probably to hypermetropia.

In early cases of presbyopia a lag of less than six-prism dioptres of exophoria is frequently found, because the hardening lens requires a greater ciliary effort to alter the curvature for near vision; the proper reading addition restores the normal physiological exophoria.

\section{Ductions}

Duction is the measure of the amplitude of fusion reserve. Duction describes the innervation which will cause the eye muscles to move in the interest of binocular vision.

First grade fusion must exist before duction can be estimated. We measure the power the eyes have of maintaining binocular vision against the diplopia producing effect of prisms.

Adduction is the amount of convergence which can be exerted irrespective of accommodational convergence.

The measurement, both for distance and near is made with baseout prisms before the eyes, geared together so that equal amounts can be rotated simultaneously. The power is increased until the image blurs, which means that accommodational convergence is called into use. This blur point measures the fusional convergence amplitude.

If prism power is added until diplopia is produced the limit of accommodational convergence has been reached.

The prisms are now reversed until the prism power recording fusion is ascertained. The recovery point is of some importance as 
insufficient convergence usually has a very low recovery point. Normally the recovery point is half the amount of prism power which produced diplopia.

Normal adduction at distance measures eighteen prism dioptres to the blur point, and twenty-four prism dioptres before diplopia occurs.

At the near point thirty prism dioptres can be overcome.

Abduction is measured with base-in prisms and since divergence cannot stimulate accommodation, the prism power causing diplopia is the measure of abduction.

Normal abduction at distance measures eight prism dioptres and at the near point eighteen prism dioptres is the measurement.

Supra and infra-ductions are measured in the same way with vertical prisms. The amplitude of supraduction measures two prism dioptres and infraduction three dioptres.

A slight degree of hyperphoria when corrected often makes the treatment of esophoria much easier. If duction reserves are normal many imbalances cause no symptoms whatever.

\section{Treatment}

The treatment of heterophoria is very briefly outlined as follows :-

Exophoria with a low adductive reserve, may be refractive or pathological. Hypermetropes with exophoria should not as a general rule be given plus lenses which increase the near point imbalance, reduced lenses will often reduce the exophoria which shows that it was of the exhaustive type.

Insufficient convergence, where the general health is not the cause of the high exophoria, responds readily to orthoptic training. Exercises with increasingly strong base-out prisms, on either the variable prism stereoscope or the synoptiscope will increase adduction amplitude, in a few weeks, and provide a reserve whereby exophoria at the near point can be overcome.

The stereoscopic sense, which is always of a low grade type, is greatly improved, and suspension of vision in one or the other eye will no longer take place.

In well established presbyopia showing a high exophoria at the near point, base-in prisms incorporated with the reading addition, will prove satisfactory.

Esophoria with a low adduction is much more unceriain in its treatment.

As, however, it is often due to hypermetropia either latent or manifest, refractive correction is all that may be needed.

When the correction eliminates the distant imbalance but has no effect on the near, a bifocal addition irrespective of age, will give comfort when reading. 
In other cases, when general pathological causes can be ruled out, orthoptic training with base-in prisms may be tried, with the hope of increasing the abduction reserve.

The wearing of permanent weak base-in prisms (which must not cause diplopia) can be prescribed at the same time.

As a temporary measure, in older patients, who have to do much close work relieving prisms (base-out) must be ordered.

\section{THE THIRD BRAZILIAN CONGRESS IN OPHTHALMOLOGY. JULY, 1939}

BY

\section{E. E. CASS \\ LONDON}

ON Wednesday, July 5th, in company with Professor Linneu Silva, Professor Moacyr Alvaro, Professor Correo Meyer and Dr. Assis Brasil, I flew from Rio de Janeiro to Bello Horizonte, the capital of Estado do Minas Gerais, where the Third Congress of Ophthalmology was being held. We had to fly high, as the country is very mountainous and our descent was too sudden to be pleasant. We were met by Professor Santa Cecilia, the President of the Society and by Dr. Hilton Rocha, the Secretary. We were awaited with some curiosity, as Professor Silva had sent a telegram

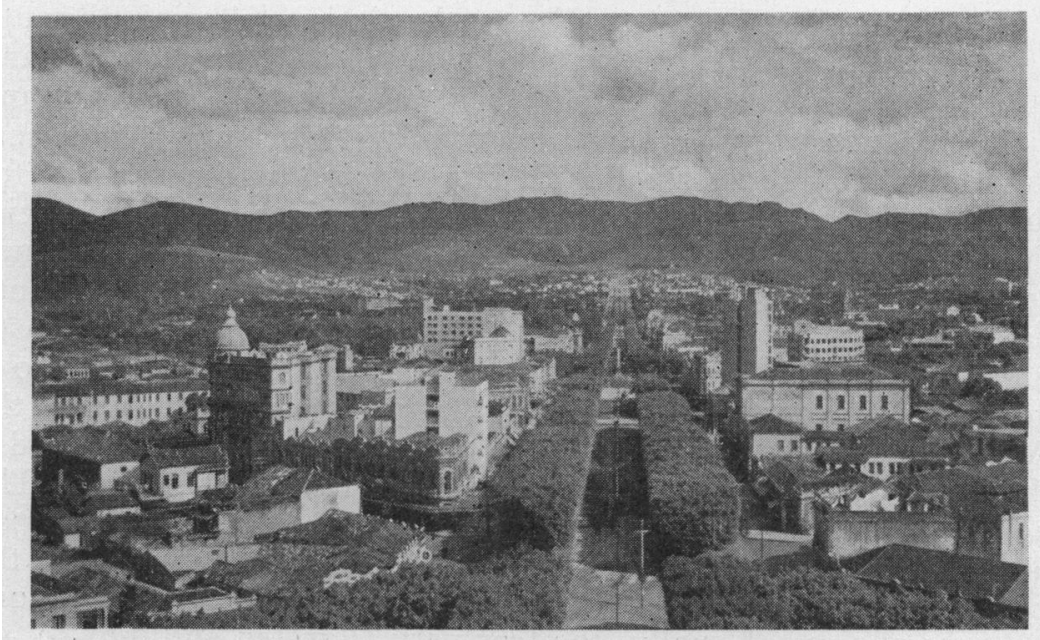

Av. Aff. Penna-Bello Horizonte. 